\title{
Research on the Knowledge Representation Method of Instance Based on Functional Surface
}

\author{
Pengjie Zhai ${ }^{\mathrm{a}}$, Changqing $\mathrm{Gao}^{\mathrm{b}^{*}}$, Xiaowen $\mathrm{Chi}^{\mathrm{c}}$ and Bo Yang ${ }^{\mathrm{d}}$ \\ School of Mechanical Engineering, University of Jinan, Jinan 250022, P.R.China \\ azhaipj2012@163.com, ${ }^{\text {b* me_gaocq@ujn.edu.cn, }{ }^{c} 18764117726 @ 163 . c o m}$ \\ dme_yangb@ujn.edu.cn
}

Keywords: Functional Surface, Assembly Relation, The Knowledge Expression, Object-oriented, Instance Retrieval.

Abstract. In this paper, in order to improve the thinking ability and get faster and better innovative ways in designing procedure, functional surface ,assembly relation and the knowledge representation method for instance is chosen as the emphasis of this paper .By studying the relationship between functional surfaces and assembly, the mapping between functional surface and the assembly relationship are determined, and the functional surface information of parts are stored in order to extract the information of functional surface. Instance is represented by object-oriented approach, and makes the instance information package easy to extract instance information, retrieval and call. The three-dimensional model and semantic information are hung together, to better stimulate the designer's creative thinking; we can get useful information from the instance information and faster find a solution.

\section{Introduction}

In 21 st century, the key to survival for the enterprises in the fierce competition is product innovation. Product innovation is the key to win for manufacturing in the market competition. If the product design is divided into product definition, conceptual design, technical design and detailed design stages, and the conceptual design stage is a closely relationship with innovation, and it is the most important aspect and the most innovative of the stage in product development process. It is the soul of the manufacturing. Conceptual design is the best stage to reflect the human creative activity in the new product development process, and it is the critical stage of product design and the most difficult stage of the design [1].

During the product design process, product design become more and more complex, how to stimulate the designer's inspiration in the design process is particularly important for the entire design process. Functional surface of parts have the larger impact with the product's function. Most of the parts have the direct or indirect relationship with two or more parts which mainly depend on the surface of the parts to realize, and these surfaces are the functional surface of parts.

In this paper, through the expression way of example, the functions and structures were hung together, and TRIZ was used into the expression instance. By this expression, it can directly affect the designer of critical thinking, and stimulate innovation and inspiration to produce.

\section{The Definition of Functional Surface}

From the perspective of geometry type, point, line, surface, body are the integral elements of spatial geometry. Point is the most basic geometric elements, but point and line are vague on the concept. Surface geometry can accurately express meaning.

Traditional way of mapping is the mapping between the function and the structures in product design. This mapping way is uncertainty, morbid, multiplicity and other issues [2]. Functional surface will be introduced as an intermediate media of the structure and function, which achieved the mapping among the function, functional surface and structure, as shown in Figure 1. 


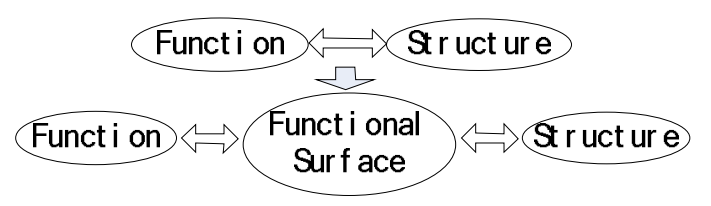

Fig. 1 The mapping among the function, functional surface and structure

Product function is rarely accomplished only by a separate part, usually through the interaction between parts to achieve. The interaction between parts is mainly the interaction result of some important surface, so that the product functions are mainly achieved by these important functional surfaces. Functional surface is the basic unit to achieve product function, but also the basic unit of product structure [3].

Parts functional surface is an important factor in determining the mechanical function, which is designed to be the core issue of component design [4]. The surface of the product is generally divided into two types: one is functional surfaces, which is closely related with product functions; the other is non-functional surface, and it has little relation with the product function.

For example the coincide of the bearing with the shaft, the bearing inner and outer race are closely related with bearing functions, but the side of bearings is related with the bearing function barely, So the functional surface of the bearing is the bearing inner and bearing outer. The shaft's functional surface is the outer surface. As shown in Figure 2.

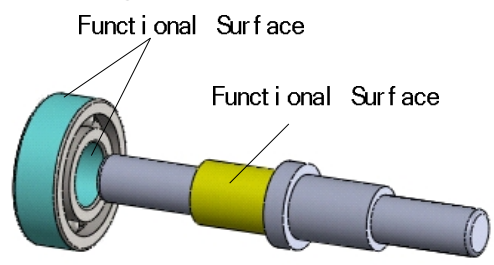

Fig. 2 The functional surfaces of the bearing and the shaft

Functional surface of products is a greater impact on the product function, but the non-functional surface just impact the construction or location of products. The non-functional surface has little effect on the product's function. So when the product structure is improved, the functional surface of the product is mainly transformed. The determination of the functional surface is the focus of the study by designers.

\section{Functional Surface Model}

Surface is the basic elements to form part shape. Parts are composed of different surfaces according to different forms (intersection, parallel, etc.). Functional surface as the minimum geometric unit with a clear functional concept has dual attributes of geometrical and function. Functional surface as an information carrier put the expression of product information on surface which is lower than the part features. That can describe product features and deliver structural information [5].

In fact, the combination of the two parts is the same definition with the combination of the functional surfaces. One part may be interacting with a number of different parts. There may be multiple functional surfaces. Functional surface can be determined by extracting shape information of parts according to the order of the combination. The functional surface of the part can be expressed as follows. FP $=\{$ FuncFace1, FuncFace2, .. FuncFacei $\}$. Functional surface can be numbered according to the assembly sequence, $F P=\{$ FuncFace1, FuncFace2, ... FuncFace $i\}=\{01,02,03,04, \ldots, i\}$. The purpose of number is to facilitate the change of functional surface properties, highlighting the functional surfaces.

Parts are A, B, C, D, E, and the combination form is represented with two letters, for example AB, $\mathrm{AC}$, and so on. The combination of the two parts is the same definition with the combination of the functional surfaces. So the form of combination between functional surface of A and functional surface of $\mathrm{B}$ is $\mathrm{AB}$. The sets of cooperative relationship of the functional surface can be expressed as follows. 
FaceRelation $=\{$ Part A, Part B, PartA.FuncFace, PartB.FuncFace, AB $\}$

Note: PartA. FuncFace - the functional surface of part A;

PartB. FuncFace - The functional surface of part B ;

$\mathrm{AB}$ - Assembly relationships between A and $\mathrm{B}$

As shown in Table 1. There are three different kinds of assembly relationships corresponding with the functional surface of the parts.

Table 1 Assembly relationships of functional surface between parts

PartA -Part B

Through object-oriented approach, it is convenient to use with the establishment of Assembly relationships classes of functional surface, and the encapsulation of functional surface relationship.

Public Class FaceRelation

Private Assem As Object

Private Part A As Object

Private Part B As Object

Private Part A.FuncFace As SldWorks.Face2

Private Part B.FuncFace As SldWorks.Face2

Private AssemRelation As String

End Class

Functional surface is the basic unit to realize the product function. The function of the product is achieved by the combination between the functional surfaces of parts. Functional surface is the expression vectors both the structure and the function. The function can be determined by the function relationship on the functional surface.

Function relationship between the functional surfaces can be expressed as follows.

FuncRelation $=\{$ Part A, Part B, PartA FuncFace, PartB. FuncFace, Function $\}$

Note: Function - the function between Part A and Part B

The definition of the relationship between functional surface and function as follows.

Public Class FuncRelation

Private Assem As Object

Private Part A As Object

Private Part B As Object

Private Part A.FuncFace As SldWorks.Face2

Private Part B.FuncFace As SldWorks.Face2

Private Function As String

End Class 


\section{The knowledge representation of instance}

Knowledge representation is a compromise transformation. It turns the actual knowledge of human experts expression into the form that machine is easy to operation. On one hand, it can reflect the original meaning of knowledge. On the other hand, it is easy to be recognized, reasoning and analysis by computer. Common knowledge expression has the facts expressions, logic expressions, production expressions, Semantic Web framework expressions, process expressions, etc. [6].

These single expressions are difficult to design a complete expression of knowledge. The object-oriented knowledge representation is a new method of knowledge representation. It made the single knowledge representation method in accordance with the principles of object-oriented programming consisting of a mixture of knowledge in the form of expression, in line with reorganization model of design objects for expert [7]. Instance Representation can be combined with TRIZ tools. We can easily organize instances, and retrieve relevant examples or best practices [8].

Instance organization expression way is as follows:

$<$ Instance information $>$ \{nstance ID, Name of the instance, Instance belongs to industry field, background knowledge, Instance problem description, TRIZ theory corresponding instances, The instance entity information $\}$

$<$ TRIZ Theory $>$ the invention principles, the separation principle, Effect library, Standard solution\}

$<$ Entity information $>$ \{Assembly, Part information, Assembly relationship between parts, parts function, Entity material, Entity tolerance, Entity motion simulation $\}$

$<$ Part Information $>$ \{Part number, Part name, Functional surfaces of part, Assembly relationships between functional surfaces $\}$

It is convenient to view through changing the structure of the assembly by different properties displayed. We should change the structure of the assembly part with different properties displayed, and then stored the assembly. When calling the assembly, you can directly see the structure to change, capture the focus of attention easily. It facilitates the entire design process.

Research on entity movement simulation:

Through macro recording, entity movement simulation program was compiled, and then put it into the built database. When the $3 \mathrm{~d}$ model is displayed, the compiled motion simulation program is called and the motion simulation animation is presented to the designers. It shows the structure and function of the product vividly, and designers can more quickly gain inspiration from the instance.

Instance can be defined using the method of object-oriented as follows.

Public Class ShiLi

Dim ID As Integer

Dim Name As String

Dim Field As String

Dim BgK As String

Dim Pd As String

Dim Ip As Integer

Dim Sp As Integer

Dim Ef As Integer

Dim Ss As Integer

Dim A As Object

Private Class Entity information A

Dim Assem As Object

Dim Part As Object

Dim PNo As Integer

Dim PName As String

DimPart.FuncFace As Object

Dim FaceRelation As String
'Instance ID

'Instance name

'Instance belongs to industry field

'Background knowledge

'Instance problem description

'The invention principles

'The separation principle

'Effect library

'Standard solution

'Entity information

'Assembly

'Part information

'Part number

'Part name

'Functional surfaces

'Assembly relationships between functional

surfaces 
Dim AssemRelation As String

Dim Function As String

Dim M As String

Dim T As Long

Dim Mo As Object
'Assembly relationship between parts

'Function

'Entity material

'Entity tolerance

'Entity motion simulation

\section{End Class}

\section{End Class}

Instance information is expressed in the object-oriented way and the property was encapsulated. It is convenient to retrieval and call for the instance. Parts information, assembly relations between parts, and assembly relationships corresponding information of functional surface can be extracted from entities information of instance by traversing the assembly model, and then analogical reasoning, to find inspiration from the instance and solve the problems which exist in the design to complete the design process.

The application process of the instance is shown in Figure 3.

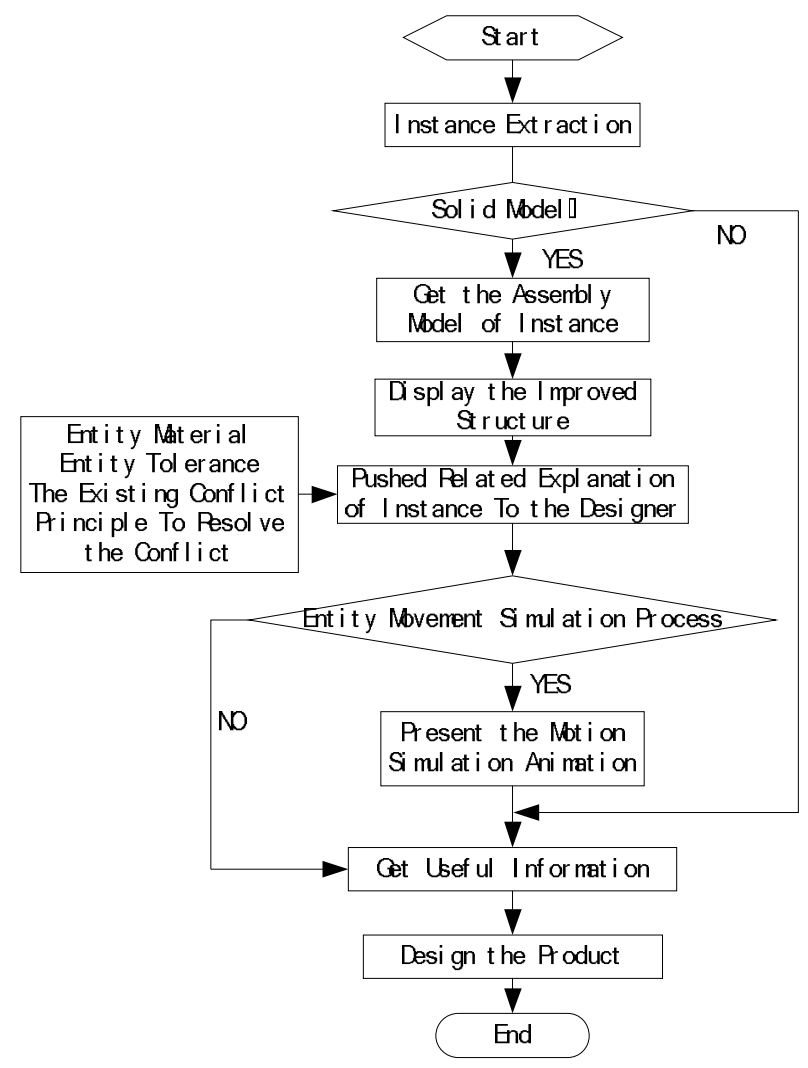

Fig. 3 The application process of the instance

The principles innovative ways can be combined with structure better, so designers can be stimulated creative thinking, get useful information from the instance information and find solutions faster by the expression in the innovation process.

Designers could get the inspiration from the instance and access the design plan faster. Not only attributed to the expression method of instance, one's own experience, knowledge and the creativity in the mind play very important effect on the whole process. The innovation process based on the instance is shown in figure 4. 


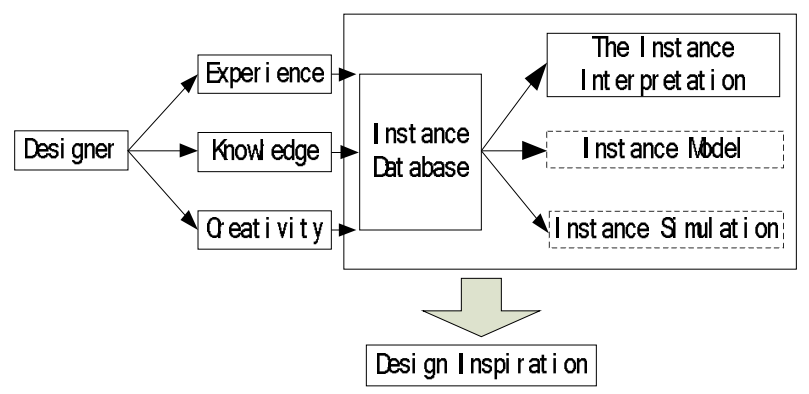

Fig. 4 The innovation process based on the instance

\section{Conclusions}

At the first half part of this paper, the establishment of function model and the determination of the functional surface of a three-dimensional model in the instance were studied. At the last part, the entire instance information was described. Based on the research of the knowledge expression method of instance, the instance information was represented through the way of object-oriented and encapsulated the attribute information in order to retrieve the instance. The knowledge representation way of instance directly affects the retrieval efficiency of the instance. The expression way of instance is the combination of three-dimensional models and semantic information together, to achieve the unity of form and meaning. In this way it can stimulate creative thinking of designers. Extraction of functional surface information, traversing the function surface, retrieving the instance and the way to push the instance in the three-dimensional modeling space should be explored on later.

\section{Acknowledgement}

This work was financially supported by Science and Technology Development Plan Project of Shan-dong Province (2014GGX106003), Large Equipment Upgrade Project of Shandong Province (2012SJGZ15) and Innovation Method Project(2013IM022300).

\section{References}

[1] Qiu Cheng, Feng Jun-wen, Gao Chang-qing, Ding Jun-wu. The Research and Developing Trend of Conceptual Design for Mechanical Product[J]. Modular machine and automation technology,2007 (10):1-4. (In Chinese)

[2] Yang Jin-yong, Huang Ke-zheng, Huo Zhi-pu. Function Surface-based Growth Design Theory[J]. Chinese journal of mechanical engineering, 2008,44(8):29-35. (In Chinese)

[3] Chen Hong-wu, Huang Ke-zheng, Yang Bo. Research and Implementation on Product Design Automation Based on Function Surface, Machine Design and Research, 2004, 20(3): 24-27. (In Chinese)

[4] Huang Chun-ying.Mechanical Creative Design[M], BeiJing:the High Education Press of China, 2000. (In Chinese)

[5] Yang Bo, Gao Chang-qing, Wang Xing-zu, Yao Kong, Yin Xiao-ling. Cell based product structure conceptual design. Computer Integrated Manufacturing System,2014,20(3): 471-485. (In Chinese)

[6] Bi Hong-li. Expression of Knowledge and Experience and Their Application in Conceptual Design. Aeronautical manufacturing technology, 2003(2):63-65. (In Chinese)

[7] Xiao Qian,Zhou Xin-jian,Deng Yi-xiong. The Research of Product Design Knowledge's Express Method Based on Object-oriented. Machine tool and hydraulics,2005 (1):171-173. (In Chinese) 
[8] Wang Ji-qing, Wen Zheng-zhong, Sun Hua-li. Research on the computer aided creative system based on TRIZ and cases. Journal of Xidian University,2003, 30(6): 827-830. (In Chinese) 